\title{
Differential impact of allograft rejection on kidney transplant patients with BK virus infection
}

\author{
Ji Won Min ${ }^{1}$, Jae Berm Park ${ }^{2}$, Jung Hwan Park ${ }^{3}$, Jong-Won Park ${ }^{4}$, Jaeseok Yang ${ }^{5}$, Curie Ahn ${ }^{6}$, Chul Woo Yang ${ }^{7}$, \\ Byung Ha Chung ${ }^{7}$

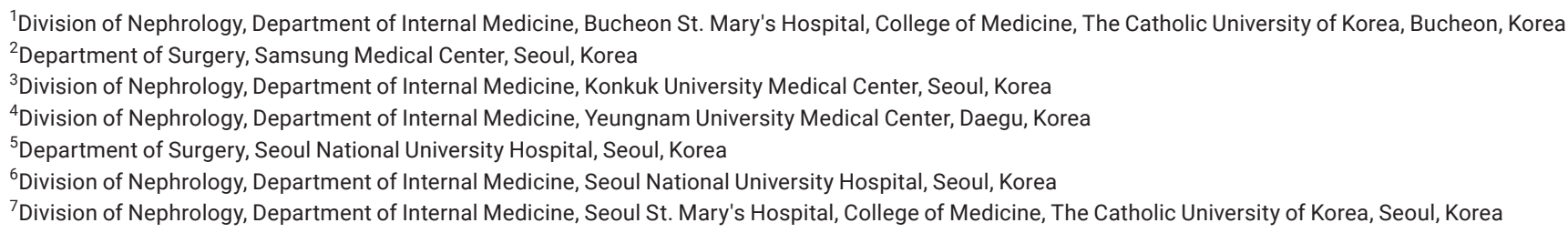

Background: BK virus-associated nephropathy (BKVAN) is a known risk factor for allograft dysfunction and graft failure in kidney transplant recipients. The mainstay of treatment for BKVAN is reduction of immunosuppression and this may increase risk for acute rejection. We proposed to observe the effects of acute rejection on patients with BKVAN.

Methods: Using data from the Korean Organ Transplantation Registry, a nationwide organ transplantation database, we compared graft function, and allograft survival in BKVAN patients with or without biopsy proven acute rejection (BPAR).

Results: Among the 5,403 patients who received kidney transplantation between 2014 and June 2019, a total of 97 patients were diagnosed with BKVAN. Twenty-six patients (27\%) developed BPAR within 6 months of BKVAN diagnosis, 71 patients did not. There were no differences in baseline characteristics, immunosuppression or BKVAN treatment methods between the BPAR and no BPAR groups. There was a significant decrease in allograft function in the BPAR group compared to the no BPAR group in both the 1-year (BPAR creatinine [Cr], $2.5 \pm 1.9 \mathrm{mg} / \mathrm{dL}$ vs. no BPAR $\mathrm{Cr}, 1.9 \pm 0.9 \mathrm{mg} / \mathrm{dL} ; \mathrm{P}=0.044$ ) and 2-year follow-up period (BPAR $\mathrm{Cr}, 3.8 \pm 3.6 \mathrm{mg} / \mathrm{dL}$ vs. no BPAR $\mathrm{Cr}, 2.2 \pm 1.0 \mathrm{mg} / \mathrm{dL} ; \mathrm{P}=0.015)$. The BPAR group had lower allograft survival rates compared to the no BPAR group, although not statistically significant $(\mathrm{P}=0.474)$. On multivariate Cox regression analysis, MMF discontinuation was observed as a significant risk factor for rejection in BKVAN patients (hazard ratio, 4.000; 95\% confidence interval [Cl], 1.014$15.775 ; \mathrm{P}=0.048$ ).

Conclusions: Acute rejection with BKVAN is associated with poorer allograft function and survival. Also, discontinuation of MMF as treatment for BKVAN increases risk for acute rejection. 